\title{
Kinematics and Dynamics Analysis of a Hybrid Parallel-Serial Micromanipulator Designed for Biomedical Applications
}

\author{
M. A. Nasseri ${ }^{1}$, M. Eder ${ }^{2}$, D. Eberts ${ }^{2}$, S. Nair ${ }^{2}$, M. Maier ${ }^{3}$, D. Zapp ${ }^{3}$, C. P. Lohmann ${ }^{3}$ and A. Knoll ${ }^{2}$
}

\begin{abstract}
This paper presents the kinematic and dynamic analysis of a robot for 6 Degrees Of Freedom (DOF) micromanipulation. This robot is designed for performing intraocular manipulation but its application is not limited to ophthalmic surgery. The novel hybrid parallel-serial mechanism designed for this robot enables microscale motions with high stiffness and sufficient output forces. This portable robot can be easily integrated into standard biomedical environments and does not require any modification of conventional surgical tools. The contribution of this work is a novel design of a miniature micromanipulator comprising piezo actuator based parallel coupled joints which allow adjustable Remote Center of Motion (RCM). The advantages of the introduced mechanism compared to similar mechanisms are compactness, stiffness and simplicity of mathematical computation.
\end{abstract}

\section{INTRODUCTION}

Ophthalmic surgery is a discipline within the medical domain that immensely depends on the skills and experiences of the surgeons. The tremor of the surgeon's hand has to be as minimal as possible. As compared to simpler ophthalmic procedures such as cataract treatment, ophthalmology frequently implies sophisticated tasks demanding high-precision maneuvers, e.g. during vitreoretinal surgeries. Vitreoretinal surgery is performed in many vitreoretinal diseases including retinal detachment, diabetic retinopathy, macular degeneration, endophthalmitis and retinal vascular occlusions.

The current problem is that there is no satisfying cure for these ophthalmic maladies, such as retinal vascular occlusion, which is based on a clot that is formed in one of the retinal arteries or veins, having a cross sectional diameter of $80 \mu \mathrm{m}$. Over 16 million people worldwide suffer from this disease today [1]. A promising treatment for this disease is the injection of clot-dissolving drugs like tissue Plasminogen Activator (tPA) directly into the affected vessel [2]. Wei et al. suggested a method using stenting as a surgical care for Central Retinal Vein Occlusion (CRVO) [3]. However, the surgeon's movement skills are not sufficient enough to perform tasks such as locating the tools and especially holding it for at least 30 seconds, since the tremor of the surgeon's hand is already in the range of $108 \mu \mathrm{m}$ [4].

\footnotetext{
*This work was supported by TUM Graduate School of Information Science in Health

${ }^{1}$ M. Ali Nasseri is with Graduate School of Information Science in Health, Technische Universität München nasseri at in.tum.de

${ }^{2}$ M. Eder, D. Eberts, S. Nair and A. Knoll are with the Department of Robotics and Embedded Systems, Institut für Informatik, Technische Universität München ederma, eberts, nair, knoll at in. tum. de

${ }^{3}$ M. Maier, D. Zapp and C. P. Lohmann are with the Augenklinik rechts der Isar, Technische Universität München mathias.maier, daniel.zapp, c.lohmann at mri.tum.de
}

Therefore, it is practically impossible for the surgeon to accomplish such a high-precision surgery. Thus, there is a need to develop a robotic assistance system that is able to overcome these current limitations and furthermore provide new surgical abilities.

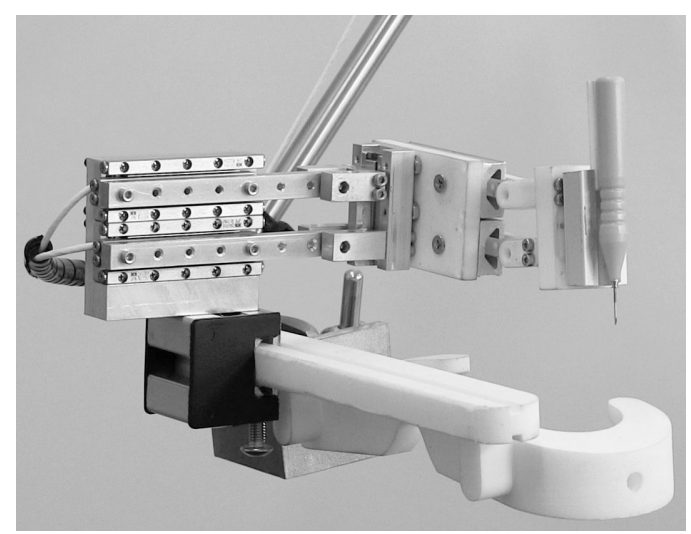

Fig. 1. 6DOF miniature robot designed for ophthalmic surgery

\section{A. Related Works}

In order to meet the biomedical application requirements, it is essential to combine both rigidity and optimal working space of a compact micromanipulator. To achieve this task, one of the most important challenge is identifying optimal kinematics. According to publications by Romdhane, Tanev or Yang et al., hybrid parallel-serial kinematics are quite promising in order to unite the advantages of both parallel and serial kinematics, in particular in terms of rigidity and working space [14], [15], [16]. In the specific domain of retinal eye surgeries, various internationally active groups have developed different systems with diverse kinematics. A first ocular robotics system was developed in 1989 by Guerrouad and Jolly, named "stereotaxical micromanipulator (SMOS)". This system was a spherical micromanipulator attached to a 3D stage with 6DOF motion [5]. Charles et al. introduced an eye surgery robot (RAMS) in 1997, a 6DOF serial manipulator in master-slave configuration with a precision of about $10 \mu \mathrm{m}$ [6]. Since 1997 a few robotic research groups are active within the domain of ophthalmic surgery. A hybrid two-armed microsurgical robot was created by Wei et al., which is based on a parallel hexapod platform [7]. A surgical tool in hand-held design was approached by Riviere's group, which offers tremor cancellation features [8]. At the Johns Hopkins University, a steady-hand system was developed as a robot assisting ophthalmic surgeons [9]. The steady-hand robot is designed 
as a cooperative surgical device to increase the precision and to filter the surgeon's hand tremor. A spherical C-arm device was proposed at Tokyo University in 2009, which is a system with comparably large dimensions [10]. Recently, researchers from Katholieke Universiteit Leuven [11] and Meenik et al. from TU Eindhoven have been active in this field. The group at TU Eindhoven came up with a fixed RCM mechanism for retinal surgery being fixed to the operation table [12].

\section{B. Limitations and Solutions}

Due to the fact that ophthalmic surgery is an area that deals with precise motions during the operation, it is evident that a robotic assistance device requires to be extremely precise $(\mu m$ scale). In order to fit into the clinical environment, the device has to meet certain demands in terms of installation space since typically there are other medical devices in close range, such as microscopes. Beside this, safety and being adaptable to conventional surgical tools are of primary importance. So far there is no available device to be deployed for practical clinical use. In this paper a robotic solution is presented to overcome the current limitations in mechanical design. A novel parallel-serial hybrid kinematics with comparably simple control algorithms is proposed to meet the demands during ophthalmic procedures. The gist of this paper is to show details of a new kinematics designed for biomedical or rather ophthalmic applications and to prove the basic ideas of an easily controllable, light-weight and compact assistance robot to be an optimal surgical device that is practically usable in the medical environment.

\section{Organization of this Paper}

The remainders of this paper is structured as follows: In section II, a description of the parallel coupled joint mechanism with its advantages is presented and followed by the serial robot configuration which consists of kinematics, singularity and dynamics analysis of the robot. In section III, performance validation of the robot is investigated. Thereafter, conclusions and future work are discussed in section IV.

\section{The Developed Robot}

In this work a robot comprising prismatic piezo actuators was designed and developed. The kinematics consists of four segments; two parallel coupled joint elements, one prismatic and one optional revolute joint in the end effector which collectively enables 6DOF tool motion.

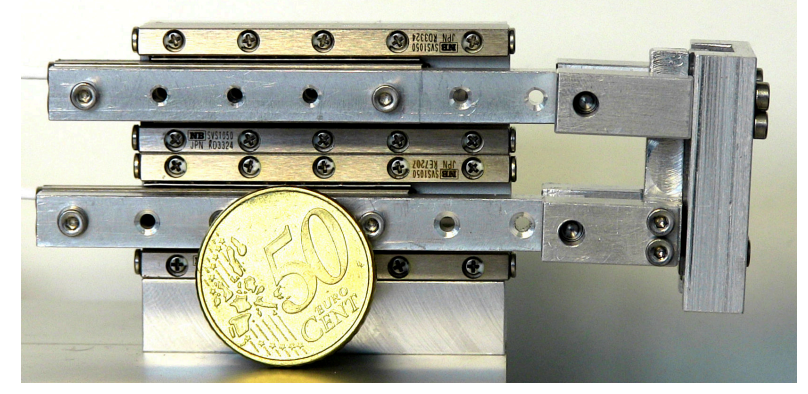

Fig. 2. Parallel Coupled Joint Mechanism (PCJM) developed to be used as the joint elements of ophthalmic surgery robot

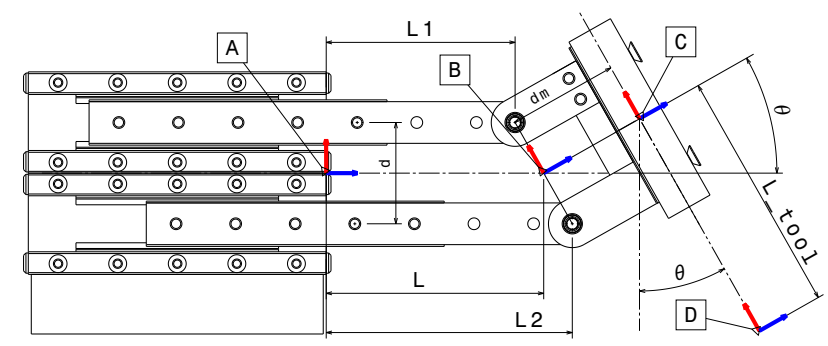

Fig. 3. Kinematics analysis of PCJM

\section{A. Parallel Coupled Joint Mechanism (PCJM)}

One novel contribution of this work is a parallel coupled joint mechanism (see Fig. 2). In this mechanism the differential displacement of two translational motions is converted to one translation and one rotation (see Fig. 3). It should be noted that $L 1$ and $L 2$ are linear displacements of each prismatic joint with the distance $d$ from each other. $L$ and $\theta$ are linear and angular displacements of the mechanism, $d_{m}$ is the length of the end effector and $L_{t o o l}$ is the length of the tool, from gripper to tool tip.

Eq.(1) represent the simplification of the mechanism by mapping $L 1$ and $L 2$ to $L$ and $\theta$, respectively

$$
L=\frac{L 1+L 2}{2}, \quad \theta=\arctan \left(\frac{L 2-L 1}{d}\right)
$$

To guarantee a $1: 1$ mapping of $L 1, L 2$ to $L$ and $\theta$, the singularities of the mapping should be analyzed. The Jacobian matrix $\left(J_{L}\right)$ is written as follows:

$$
\begin{gathered}
{\left[\begin{array}{l}
q_{1} \\
q_{2}
\end{array}\right]=f\left[\begin{array}{l}
L_{1} \\
L_{2}
\end{array}\right], \quad q_{1}=L \text { and } q_{2}=\theta} \\
\dot{q}=\overbrace{\frac{\partial f(L)}{\partial L}}^{J_{L}} \dot{L} \\
{\left[\begin{array}{c}
\dot{q}_{1} \\
\dot{q_{2}}
\end{array}\right]=\left[\begin{array}{cc}
\frac{1}{2} & \frac{1}{2} \\
\frac{-d}{d^{2}+\left(L_{2}-L_{1}\right)^{2}} & \frac{d}{d^{2}+\left(L_{2}-L_{1}\right)^{2}}
\end{array}\right]\left[\begin{array}{c}
\dot{L_{1}} \\
\dot{L_{2}}
\end{array}\right]}
\end{gathered}
$$

It can be observed that the determinant of the Jacobian matrix $\left(J_{L}\right)$ is 0 , only if $d=0$ which implies that for the proposed configuration, where the distance of two prismatic parallel actuators is more than $0, J_{L}$ matrix is full rank. Therefore, there are no singularities or in other words for any $L 1$ and $L 2$ a unique $L$ and $\theta$ exists and vice versa.

Forward Kinematics: Now, with this assumption any parallel coupled joint simplifies to a prismatic and a revolute joint in serial. For instance, the simplified homogeneous transformation matrix of point $\mathrm{B}$ in Fig. 3 with respect to point A is:

$$
T_{A}^{B}=\left[\begin{array}{cccc}
\cos \theta & -\sin \theta & 0 & L \\
\sin \theta & \cos \theta & 0 & 0 \\
0 & 0 & 1 & 0 \\
0 & 0 & 0 & 1
\end{array}\right]
$$

and by considering $T_{A}^{D}=T_{A}^{B} T_{B}^{C} T_{C}^{D}$, for this configuration the homogeneous transformation matrix of the tool tip (D) with respect to the base (A) is: 


$$
T_{A}^{D}=\left[\begin{array}{cccc}
\cos \theta & -\sin \theta & 0 & L-L_{\text {tool }} \sin \theta+d_{m} \cos \theta \\
\sin \theta & \cos \theta & 0 & L_{\text {tool }} \cos \theta+d_{m} \sin \theta \\
0 & 0 & 1 & 0 \\
0 & 0 & 0 & 1
\end{array}\right]
$$

The homogeneous transformation matrices is simplified by substituting $\theta$ and $L$ by $L_{1}$ and $L_{2}$. The substitution procedure is eased using trigonometric identities.

$$
\begin{aligned}
& \cos \left(\arctan \left(\frac{L_{2}-L_{1}}{d}\right)\right)=\frac{1}{\sqrt{1+\frac{\left(L_{2}-L_{1}\right)^{2}}{d^{2}}}} \\
& \sin \left(\arctan \left(\frac{L_{2}-L_{1}}{d}\right)\right)=\frac{L_{2}-L_{1}}{d \sqrt{1+\frac{\left(L_{2}-L_{1}\right)^{2}}{d^{2}}}}
\end{aligned}
$$

With these assumptions, configurations consisting of PCJM can be simplified and analyzed as a simple serial manipulator.

Inverse Kinematics: The inverse kinematic equations of a PCJM (for the point B) in Fig. 3 is:

$$
L 1=L+\frac{d}{2} \sin (\theta), L 2=L-\frac{d}{2} \sin (\theta)
$$

1) Semi-mechanical Variable RCM Determination: For some medical and surgical applications it is needed to pivot the tool around a certain point (e.g. pivoting laprascopic tools around incision points). By taking the forward kinematics equations from Matrix (4), the 2D position of Point D $\left(X_{D}, Y_{D}\right)$ in Fig. 3 and solving Eq.(7) a unique dependency of $\mathrm{L}, \theta$ and $L_{\text {tool }}$ is observed.

$$
\left[\begin{array}{c}
L-L_{\text {tool }} \sin \theta+d_{m} \cos \theta \\
L_{\text {tool }} \cos \theta+d_{m} \sin \theta \\
0 \\
1
\end{array}\right]=\left[\begin{array}{c}
X_{D} \\
Y_{D} \\
0 \\
1
\end{array}\right]
$$

This dependency enables pivoting the tool around point D as a Remote Center of Motion (RCM). In L1 and L2 domain, this situation is interpreted as the dependent differential displacement of two parallel prismatic joints. Changing the RCM during the motion is also possible.

2) Advantages of PCJM over Serial Prismatic and Revolute Joint Configuration: As described in the previous paragraphs, the PCJM is similar to a serial pair of prismaticrevolute joints. The advantages of this mechanism over standard serial prismatic-revolute configuration are as follows:

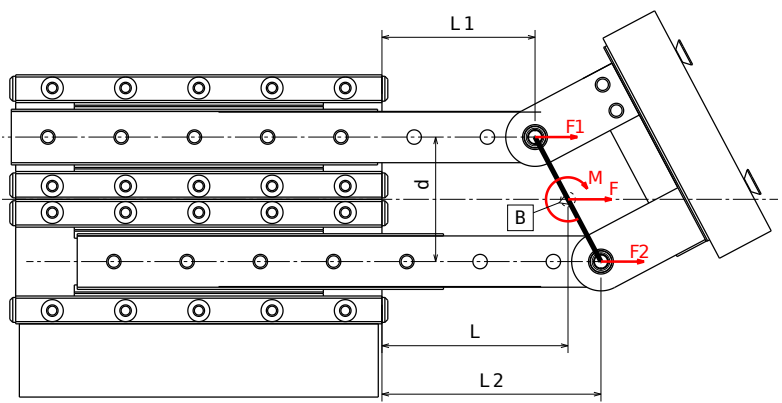

Fig. 4. Statics analysis of PCJM
Stiffness: Evidently, the parallel pair provides higher stiffness of the end effector against mechanical disturbances than a serial configuration [14], [15], [16].

Precision: Piezo prismatic actuators are among the most accurate available actuators. In case of the stages used here $50 \mathrm{~nm}$ step width is guaranteed [17].

Output force: Fig. 4 presents the static forces of PCJM. The total force which is applied to point B is $F=F 1+F 2$ and the moment applied to that point is $M=\frac{\vec{d} \times(\overrightarrow{F 1}-\overrightarrow{F 2})}{2}$. Therefore, both actuators apply forces and moments to point B. For a similar performance with classic serial prismatic-rotational configuration a prismatic actuator with output force of $>F$ and rotational actuator with the moment of $>M$ is needed (in classic serial configuration the joint $n$ should carry the weight of joint $n+1$ ). Clearly, for producing such force and moment mass is needed. Therefore, the proposed mechanism can be built more compact than a serial pair which applies the same force and moment.

Adjustable RCM: Any physical and virtual point that is in angular reach of the system can be defined as the pivoting point of the end effector or attached tool. This point can be changed or moved during motion.

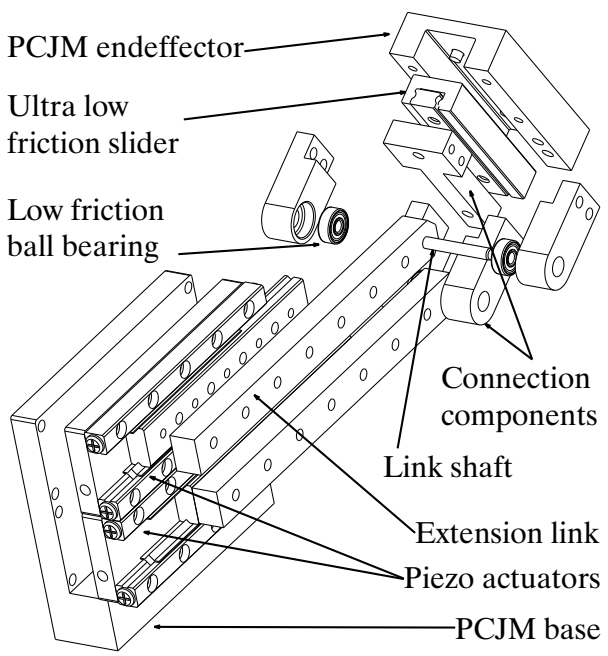

Fig. 5. Mechanical parts of PCJM

3) Developed Joint: A parallel coupled joint with piezo electric actuators ${ }^{1}$, low friction, precise mechanical components (see Fig. 5), sub $\mu m$ optical encoders and customized controller was developed (see Fig. 2). As it can be seen in Fig. 5, the length change between the tip of the two prismatic actuators was covered by using a low friction and precise micro slider. The properties of this mechanism were measured to be as follows: dimensions: $94 \pm 28 \times$ $33.5 \times 18.5 \mathrm{~mm}$, weight: $150 \mathrm{~g}$, linear travel range: $\pm 28 \mathrm{~mm}$, angular rotation: $\pm 58.734^{\circ}$, linear precision: $1 \mu \mathrm{m}$, angular precision: $3.369 \times 10^{-3 \circ}$, maximum output force: $4.97 \mathrm{~N}$, maximum linear velocity: $17.5 \frac{\mathrm{mm}}{\mathrm{s}}$ and maximum angular velocity: $63.56_{\frac{\circ}{s}}^{\circ}$.

${ }^{1}$ SmarAct linear positioners SLC-1750, SmarAct GmbH, Germany 


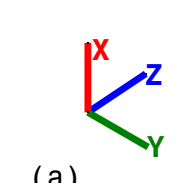

(a)

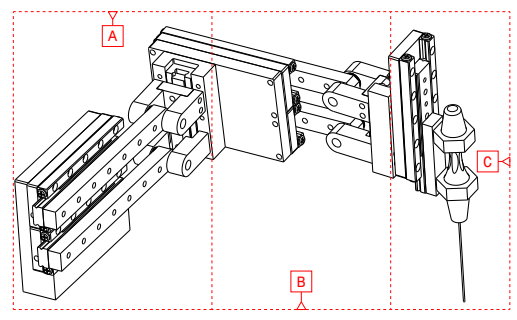

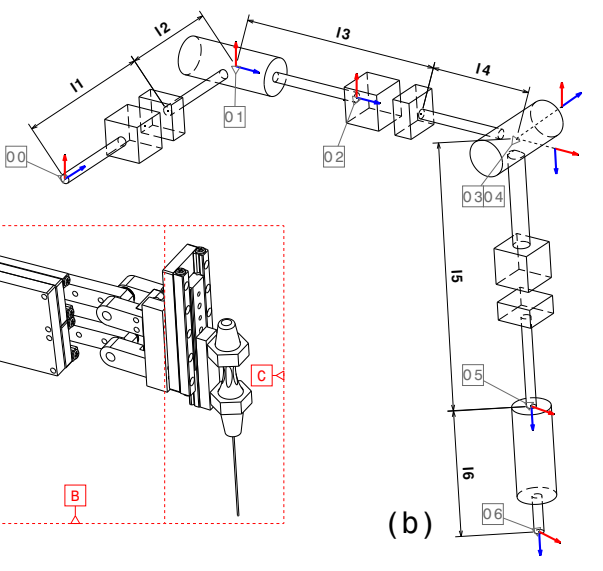

Fig. 6. (a) Serial robot: A and B are parallel coupled joint elements. C is the tool gripper consisting of a prismatic actuator and an optional tool rotator, (b) relevant and simplified model of the serial robot

\section{B. Serial Robot}

Fig. 6.(a) represents a serial configuration to perform 6 DOF manipulation. This robot consists of 3 serial segments; two PCJMs, the tool gripper comprising a prismatic actuator for tool translation, and an optional revolute actuator for tool rotation. In the previous subsection it was proved that the parallel coupled joint can be represented by a prismatic and a revolute joint to simplify the analysis. In Fig. 6.(b) the simplified model of the serial robot is shown.

Based on the simplified model, the relevant DenavitHartenberg parameters of the robot are written in Tab. I. By means of this model and the parameter information, the robot is analyzed as a standard serial manipulator.

TABLE I

DENAVIT-HARTENBERG PARAMETERS OF THE SERIAL ROBOT 0

\begin{tabular}{c|cccc} 
Link & $\theta_{i}$ & $d_{i}$ & $a_{i}$ & $\alpha_{i}$ \\
\hline 1 & 0 & $l_{1}+l_{2} \pm q_{1}$ & 0 & $\frac{-\pi}{2}$ \\
2 & $\pm q_{2}$ & $l_{3}$ & 0 & 0 \\
3 & 0 & $l_{4} \pm q_{3}$ & 0 & $\frac{+\pi}{2}$ \\
4 & $\frac{\pi}{2} \pm q_{4}$ & 0 & 0 & $\frac{-\pi}{2}$ \\
5 & 0 & $l_{5} \pm q_{5}$ & 0 & 0 \\
6 & $\pm q_{6}$ & $l_{6}$ & 0 & 0
\end{tabular}

1) Forward Kinematics: two methods can be used to derive the robot's homogeneous transformation matrices and consequently the robot's forward kinematics, either the conventional Denavit-Hartenberg method or a geometric method. By considering individual PCJM segments, the geometric method is described here: $T_{A}^{D}$ from Eq. (4) represents homogeneous transformation matrix of one PCJM. The robot's PCJMs are mounted directly on each other's end effectors (see Fig. 6.(b)). $T_{E}^{H}$ is the homogeneous transformation matrix for the second PCJM while $E, F, G, H$ are coordinate frames corresponding to $A, B, C, D$ in the first PCJM.

Taking the above considerations into account, the serial configuration consists of (1) the first PCJM $\left(T_{A}^{D}\right)$, while its $L_{\text {tool }}$ is 0 , (2) the second PCJM $\left(T_{E}^{H}\right)$ which is mounted on the first PCJM with 90 degrees of rotation around $y$ axis $\left(R_{y}\left(\frac{\pi}{2}\right)\right)$ and (3) a translational actuator $\left(T_{\text {end }}\right)$ which is mounted on second PCJM. The final homogeneous transformation $T_{A}^{e e}$ of this configuration can be written as:

$$
\begin{gathered}
T_{A}^{e e}=T_{A}^{D} \cdot R_{y}\left(\frac{\pi}{2}\right) \cdot T_{E}^{H} \cdot T_{\text {end }} \\
T_{A}^{D}=\left[\begin{array}{cccc}
\cos \theta_{a} & -\sin \theta_{a} & 0 & L_{a}+d_{m} \cos \theta_{a} \\
\sin \theta_{a} & \cos \theta_{a} & 0 & d_{m} \sin \theta_{a} \\
0 & 0 & 1 & 0 \\
0 & 0 & 0 & 1
\end{array}\right] \\
T_{E}^{H}=\left[\begin{array}{ccccc}
\cos \theta_{b} & -\sin \theta_{b} & 0 & L_{b}-L_{\text {tool }} \sin \theta_{b}+d_{m} \cos \theta_{b} \\
\sin \theta_{b} & \cos \theta_{b} & 0 & L_{\text {tool }} \cos \theta_{b}+d_{m} \sin \theta_{b} \\
0 & 0 & 1 & 0 \\
0 & 0 & 0 & 1
\end{array}\right] \\
R_{y}\left(\frac{\pi}{2}\right)=\left[\begin{array}{cccc}
0 & 0 & -1 & 0 \\
0 & 1 & 0 & 0 \\
1 & 0 & 0 & 0 \\
0 & 0 & 0 & 1
\end{array}\right], \quad T_{\text {end }}=\left[\begin{array}{cccc}
1 & 0 & 0 & 0 \\
0 & 0 & 1 & L 5 \\
0 & -1 & 1 & 0 \\
0 & 0 & 0 & 1
\end{array}\right]
\end{gathered}
$$

By using Eq. (1) the parameters: $\theta_{a}, \theta_{b}, L_{a}$ and $L_{b}$ can be mapped to $L 1, L 2, L 3$ and $L 4$. Thereafter, homogeneous transformation and forward kinematics of the robot in $L$ domain is observed.

2) Inverse kinematics: Evaluating the forward kinematics given in Equation (8) yields:

$$
T_{A}^{e e}=\left[\begin{array}{ll}
R & p \\
0 & 1
\end{array}\right]
$$

with $R \in S O(3)$ being a rotation matrix representing the end effector's orientation and $p \in \mathbb{R}^{3}$ being a vector representing the end effector's position. For $p:=\left[p_{x}, p_{z}, p_{y}\right]^{T}$ we therefore have:

$$
\begin{aligned}
p_{x} & =L_{a}+d_{m} \cos \left(\theta_{a}\right)-\sin \left(\theta_{a}\right) \cdot\left[L_{\text {tool }} \cos \left(\theta_{b}\right)+d_{m} \sin \left(\theta_{b}\right)\right] \\
& -L 5 \cdot \sin \left(\theta_{a}\right) \sin \left(\theta_{b}\right) \\
p_{z} & =L 5 \cdot \cos \left(\theta_{a}\right) \cos \left(\theta_{b}\right)+\cos \left(\theta_{a}\right) \cdot\left[L_{t o o l} \cos \left(\theta_{b}\right)+d_{m} \sin \left(\theta_{b}\right)\right] \\
& +d_{m} \sin \left(\theta_{a}\right) \\
p_{y} & =L_{b}-L_{\text {tool }} \sin \left(\theta_{b}\right)+d_{m} \cos \left(\theta_{b}\right)-L 5 \cdot \sin \left(\theta_{b}\right)
\end{aligned}
$$

Because the robot actuates only two angles and the inertial coordinate frame is aligned such that $\theta_{a}$ is the only angle that affects the tool's orientation about the inertial frame's y-axis, it is reasonable to suspect that the PCJM's joint angles can be retrieved easily.

Let $\left[\phi_{x}, \phi_{y}, \phi_{z}\right]^{T}$ be the orientation of the end effector. As already mentioned, one of these orientation angles is identical with joint A's angle, $\theta_{a}$. The other two angles result from $\theta_{b}$ and the rotation of joint $\mathrm{B}$ about the $\mathrm{y}$-axis:

$$
\left[\begin{array}{c}
\phi_{x} \\
\phi_{y} \\
\phi_{z}
\end{array}\right]=\left[\begin{array}{c}
\theta_{b} \sin \left(\theta_{a}\right) \\
\theta_{a} \\
\theta_{b} \cos \left(\theta_{a}\right)
\end{array}\right]
$$

We therefore have

$$
\begin{aligned}
\phi_{y} & =\theta_{a} \\
\phi_{z} & =\theta_{b} \cos \left(\theta_{a}\right)
\end{aligned}
$$

for the end effector's orientation about the $y$ - and z-axis

$$
\begin{aligned}
\theta_{a} & =\phi_{y} \\
\theta_{b} & =\frac{\phi_{y}}{\cos \left(\phi_{z}\right)}
\end{aligned}
$$

are the PCJM's angles $\theta_{a}$ and $\theta_{b}$. We can now solve Eq. (10) for $L_{a}, L_{b}$ and $L 5$ : 


$$
\begin{aligned}
& L 5=-\frac{L_{\text {tool }} \cos \left(\theta_{a}\right) \cos \left(\theta_{b}\right)+\sin \left(\theta_{b}\right) \cos \left(\theta_{a}\right)+d_{m} \sin \left(\theta_{a}\right)-p_{z}}{\cos \left(\theta_{a}\right) \cos \left(\theta_{b}\right)} \\
& L_{a}=L_{\text {tool }} \sin \left(\theta_{a}\right) \cos \left(\theta_{b}\right)+L 5 \cdot \sin \left(\theta_{a}\right) \sin \left(\theta_{b}\right) \\
& +d_{m}\left[\sin \left(\theta_{a}\right) \sin \left(\theta_{b}\right)-\cos \left(\theta_{a}\right)\right]+p_{x} \\
& L_{b}=L_{\text {tool }} \sin \left(\theta_{b}\right)-d_{m} \cos \left(\theta_{b}\right)+L 5 \cdot \sin \left(\theta_{b}\right)+p_{y}
\end{aligned}
$$

In a real implementation, angles have to be determined first, then $L 5$ followed by $L_{a}$ and $L_{b}$. From Eq. (1) we know how to calculate a PCJM's position $L$ and angle $\theta$, given its prismatic joint positions $L 1$ and $L 2$. The joint positions $L 1$ and $L 2$ can conversely be determined from $L$ and $\theta$ by solving Eq. (1) for $L 1$ and $L 2$ :

$$
\begin{aligned}
& L 1=\frac{d \cdot \tan (\theta)}{2}+L \\
& L 2=2 \cdot L-L 1
\end{aligned}
$$

3) Singularities: To observe singularities of the robot, the determinant of Jacobian matrix should be analysed:

$$
\begin{gathered}
J=\left[\begin{array}{cccc}
0 & \cos \left(\theta_{b}\right) \sin \left(\theta_{a}\right)\left(L_{5}\right) & 0 & \cos \left(\theta_{a}\right) \sin \left(\theta_{b}\right)\left(L_{5}\right) \\
0 & 0 & 1 & -\cos \left(\theta_{b}\right)\left(L_{5}\right) \\
1 & \cos \left(\theta_{a}\right) \cos \left(\theta_{b}\right)\left(L_{5}\right) & 0 & 0 \\
0 & 0 & 0 & -\sin \left(\theta_{a}\right) \sin \left(\theta_{b}\right)\left(L_{5}\right) \\
0 & 1 & 0 & \sin \left(\theta_{a}\right) \\
0 & 0 & 0 & \cos \left(\theta_{a}\right) \\
-\cos \left(\theta_{a}\right) \cos \left(\theta_{b}\right) & 0 \\
-\sin \left(\theta_{b}\right) & 0 \\
\cos \left(\theta_{b}\right) \sin \left(\theta_{a}\right) & 0 \\
0 & -\cos \left(\theta_{a}\right) \cos \left(\theta_{b}\right) \\
0 & -\sin \left(\theta_{b}\right) \\
0 & \cos \left(\theta_{b}\right) \sin \left(\theta_{a}\right)
\end{array}\right]
\end{gathered}
$$

and we have the determinant as:

$$
\operatorname{det}(J)=\cos \left(\theta_{a}\right)^{3} \cos \left(\theta_{b}\right)^{2}+\cos \left(\theta_{a}\right) \cos \left(\theta_{b}\right)^{2} \sin \left(\theta_{a}\right)^{2}
$$

This determinant is zero, only if $\theta_{a}=2 k \pi$ or $\theta_{b}=2 k \pi$ which for this robot is an impossible situation to be in. Therefore, this robot has no singularities in its working space.

\section{Dynamics of the Robot}

To derive the dynamic equations of the robot, its joint space is chosen as generalized coordinates. Therefore, $q=$ $[L 1, L 2, L 3, L 4, L 5]^{T}$, a vector of all joint positions is a vector of generalized coordinates.

The aim is to derive a description of the second PCJM that is rotated by 90 degrees about the $y$-axis with respect to the first PCJM.

e robot's dynamics as a second-order mechanical system of the form

$$
M(q) \ddot{q}+C(q, \dot{q}) \dot{q}+g(q)=u
$$

where $M(q)$ is the system's mass matrix, $C(q, \dot{q})$ are the centrifugal and Coriolis terms and $g(q)$ represents potential energy terms. $u$ is the influence of external forces and moments, translated into generalized coordinates, e.g. as shown above. To examine free motions of the system, $u$ is set 0 .
The terms $C(q, \dot{q})$ can be derived from $M(q)$ by determining the mass matrix's Christoffel symbols and $g(q)$ is constructed by determining all partial derivatives of some potential function $U(x)$ w.r.t. the generalized coordinates $q$. Usually, this is the influence of gravity. The robot's kinetic energy is given by:

$$
E_{k i n}=\frac{1}{2} \dot{q}^{T} M(q) \dot{q}
$$

Therefore, determining the mass matrix is basically the same as determining the kinetic energy which is composed of all linear and angular velocities that occur in the system. The PCJMs contribute a linear and an angular component respectively. The final single prismatic joint separately contributes a linear component.

\section{The Mass Matrix}

In total, the following parameters of the robot are required and thereby have been computed:

- The masses $m_{b}, m_{c}$ and $m_{E}$ of the second PCJM, the single prismatic joint, the end effector and their respective centers of gravity (Those are needed to determine $g(q)$ ).

- The inertia tensors $I_{K b}$ and $I_{K c}$ of the second PCJM's base and the single prismatic joint's base, aligned along their respective bodies' principal axes, i.e.

$$
I_{K b}=\left[\begin{array}{ccc}
I_{b x} & 0 & 0 \\
0 & I_{b y} & 0 \\
0 & 0 & I_{b z}
\end{array}\right], \quad I_{K c}=\left[\begin{array}{ccc}
I_{c x} & 0 & 0 \\
0 & I_{c y} & 0 \\
0 & 0 & I_{c z}
\end{array}\right]
$$

With the following translational Jacobians:

$$
\begin{aligned}
J_{T a} & =\left[\begin{array}{ccccc}
1 / 2 & 1 / 2 & 0 & 0 & 0 \\
0 & 0 & 0 & 0 & 0 \\
0 & 0 & 0 & 0 & 0 \\
0 & 0 & 0 & 0 & 0 \\
0 & 0 & 0 & 0 & 0
\end{array}\right], \\
J_{T b} & =\left[\begin{array}{ccccc}
0 & 0 & 0 & 0 & 0 \\
0 & 0 & 0 & 0 & 0 \\
1 / 2 & 1 / 2 & 0 & 0 & 0 \\
0 & 0 & 0 & 0 & 0 \\
0 & 0 & 0 & 0 & 0
\end{array}\right], \quad J_{T c}=\left[\begin{array}{lllll}
0 & 0 & 0 & 0 & 0 \\
0 & 0 & 0 & 0 & 0 \\
0 & 0 & 0 & 0 & 0 \\
0 & 0 & 0 & 0 & 0 \\
0 & 0 & 0 & 0 & 1
\end{array}\right],
\end{aligned}
$$

and the following rotational Jacobians (mapping from $[L 1, L 2, L 3, L 4, L 5]$ to $\left.\left[L_{a}, \theta_{a}, L_{b}, \theta_{b}, L 5\right]\right)$ :

$$
\begin{aligned}
& J_{R a}=\left[\begin{array}{ccccc}
0 & 0 & 0 & 0 & 0 \\
-\frac{1}{d \rho_{12}^{2}} & \frac{1}{d \rho_{12}^{2}} & 0 & 0 & 0 \\
0 & 0 & 0 & 0 & 0 \\
0 & 0 & 0 & 0 & 0 \\
0 & 0 & 0 & 0 & 0
\end{array}\right], \\
& J_{R b}=\left[\begin{array}{ccccc}
0 & 0 & 0 & 0 & 0 \\
0 & 0 & 0 & 0 & 0 \\
0 & 0 & 0 & 0 & 0 \\
-\frac{1}{d \rho_{34}^{2}} & \frac{1}{d \rho_{34}^{2}} & 0 & 0 & 0 \\
0 & 0 & 0 & 0 & 0
\end{array}\right]
\end{aligned}
$$

where, $\rho_{12}$ and $\rho_{34}$ are defined as in Eq. (23):

$$
\rho_{i j}:=\sqrt{1+\frac{(L j-L i)^{2}}{d^{2}}}
$$


For $L 3$ and $L 4$, the mass matrix can be constructed as follows:

$$
\begin{aligned}
M(q) & =m_{b}\left(J_{T a}^{T} J_{T a}\right)+J_{R a}^{T}\left(\mathbb{I}_{a}\right) J_{R a}^{T} \\
& +m_{c}\left(J_{T b}^{T} J_{T b}\right)+J_{R b}^{T}\left(\mathbb{I}_{b}\right) J_{R b} \\
& +m_{E}\left(J_{T c}^{T} J_{T c}\right)
\end{aligned}
$$

The result is:

$$
M(q)=\left[\begin{array}{ccccc}
M_{11} & M_{11} & 0 & 0 & 0 \\
M_{11} & M_{11} & 0 & 0 & 0 \\
0 & 0 & M_{33} & M_{34} & 0 \\
0 & 0 & M_{43} & M_{44} & 0 \\
0 & 0 & 0 & 0 & m_{E}
\end{array}\right]
$$

where, the matrix elements $M_{i j}$ are

$$
\begin{aligned}
& M_{11}=M_{22}=\frac{1}{4} m_{b}+\frac{\frac{\left(L_{1}-L_{2}\right)^{2} I_{b x}}{d^{2} \rho_{12}^{2}}+\frac{I_{b y}}{\rho_{12}^{2}}}{d^{2} \rho_{12}^{4}} \\
& M_{12}=M_{21}=\frac{1}{4} m_{b}-\frac{\frac{\left(L_{1}-L_{2}\right)^{2} I_{b x}}{d^{2} \rho_{12}^{2}}+\frac{I_{b y}}{\rho_{12}^{2}}}{d^{2} \rho_{12}^{4}} \\
& M_{33}=M_{44}=\frac{1}{4} m_{c}+\frac{\frac{\left(L_{1}-L_{2}\right)^{2} I_{c y}}{d^{2} \rho_{12}^{2}}+\frac{\left(L_{3}-L_{4}\right)^{2} I_{c x}}{d^{2} \rho_{12}^{2} \rho_{34}^{2}}+\frac{I_{c z}}{\rho_{12}^{2} \rho_{34}^{2}}}{d^{2} \rho_{34}^{4}} \\
& M_{34}=M_{43}=\frac{1}{4} m_{c}-\frac{\frac{\left(L_{1}-L_{2}\right)^{2} I_{c y}}{d^{2} \rho_{12}^{2}}+\frac{\left(L_{3}-L_{4}\right)^{2} I_{c x}}{d^{2} \rho_{12}^{2} \rho_{34}^{2}}+\frac{I_{c z}}{\rho_{12}^{2} \rho_{34}^{2}}}{d^{2} \rho_{34}^{4}}
\end{aligned}
$$

and the configuration-dependent inertia tensors $\mathbb{I}_{a}$ and $\mathbb{I}_{b}$ are:

$$
\mathbb{I}_{a}=R\left(\theta_{a}\right) I_{K a} R\left(\theta_{a}\right)^{T}, \quad \mathbb{I}_{b}=R\left(\theta_{a}, \theta_{b}\right) I_{K b} R\left(\theta_{a}, \theta_{b}\right)^{T}
$$

The rotation matrices $R\left(\theta_{a}\right)$ and $R\left(\theta_{a}, \theta_{b}\right)$ are known from the forward kinematics:

$$
\begin{aligned}
R\left(\theta_{a}, \theta_{b}\right) & =\left[\begin{array}{ccc}
-\frac{\left(L_{3}-L_{4}\right)\left(L_{1}-L_{2}\right)}{d^{2} \rho_{12} \rho_{34}} & \frac{1}{\rho_{12}} & \frac{L_{1}-L_{2}}{d \rho_{12} \rho_{34}} \\
-\frac{L_{3}-L_{4}}{d \rho_{12} \rho_{34}} & -\frac{L_{1}-L_{2}}{d \rho_{12}} & \frac{1}{\rho_{12} \rho_{34}} \\
\frac{1}{\rho_{34}} & 0 & \frac{L_{3}-L_{4}, f}{d \rho_{34}}
\end{array}\right] \\
R\left(\theta_{a}\right) & =\left[\begin{array}{ccc}
\frac{1}{\rho_{12}} & \frac{L_{1}-L_{2}}{d \rho_{12}} & 0 \\
-\frac{L_{1}-L_{2}}{d \rho_{12}} & \frac{1}{\rho_{12}} & 0 \\
0 & 0 & 1
\end{array}\right]
\end{aligned}
$$

Their use is to rotate the configuration-independent inertia matrices $I_{K a}$ and $I_{K b}$ into the current (angular) configuration and thus constructing the configuration-dependent inertia tensors.

\section{E. The Centrifugal and Coriolis Terms}

The centrifugal and Coriolis terms can be written in terms of Christoffel symbols:

$$
\begin{aligned}
C(q, \dot{q}) \dot{q} & =\left[c_{1}(q, \dot{q}), \ldots, c_{n}(q, \dot{q})\right]^{T}, \\
c_{i}(q, \dot{q}) & =\sum_{j=1}^{n}\left(\sum_{k=1}^{n} \Gamma_{i j}^{k}(q) \dot{q}_{j} \dot{q}_{k}\right)
\end{aligned}
$$

with $\dot{q}_{k}=\dot{L}_{k}, n=5$ in our case and $\Gamma_{j k}^{i}(q)$ being defined as follows:

$$
\Gamma_{j k}^{i}(q)=\frac{1}{2}\left(\frac{\partial M_{i j}(q)}{\partial q_{k}}+\frac{\partial M_{i k}(q)}{\partial q_{j}}-\frac{\partial M_{k j}(q)}{\partial q_{i}}\right)
$$

Since $M(q)$ is symmetric, these can be simplified to

$$
\Gamma_{j k}^{i}(q)=\frac{\partial M_{i j}(q)}{\partial q_{k}}-\frac{1}{2} \frac{\partial M_{k j}(q)}{\partial q_{i}}
$$

\section{F. Gravity Influence}

In the inertial frame, the gravity vector is defined as $\vec{g}=$ $\left[0,-g_{0}, 0\right]^{T}$. The base of the second PCJM is located at the end effector of the first PCJM and therefore not identical to the origin of Frame D because of its dimensions as shown in Fig. 2. As stated before, its mass is $m_{b}$. In Fig. 4, it can be seen that $L_{t o o l}$ is 0 and that the second PCJM's X-axis is aligned with the first PCJM's z-axis. Therefore, the only displacement that needs to be considered is the distance $d_{b}$ between the first PCJM's end effector's position along the $\mathrm{X}$-axis (of the first PCJM) and the second PCJM's center of gravity (as seen from the first PCJM's end effector). From kinematics, the first PCJM end effector's position is known. Modifying it, by adding said displacement $d_{b}$ :

$$
\left[\begin{array}{c}
\frac{1}{2} L_{1}+\frac{1}{2} L_{2}-\frac{d_{m}+d_{b}}{\rho_{12}} \\
\left(d_{m}+d_{b}\right) \frac{L_{2}-L_{1}}{d \rho_{12}} \\
0
\end{array}\right]
$$

Gravity only affects the y-component of this vector, which leads to the first part of the potential energy function $U$ that is required to determine the effect of gravity on the system:

$$
m_{b} g_{0}\left(d_{m}+d_{b}\right) \frac{L_{2}-L_{1}}{d \rho_{12}}
$$

With $d_{c}$ being the displacement of the single prismatic joint's base w.r.t. the second PCJM's end effector, the position of its center of gravity is

$$
\left[\begin{array}{c}
\frac{1}{2} L_{1}+\frac{1}{2} L_{2}+\frac{\left(d_{m}+d_{c}\right)}{\rho_{12}}-\frac{\left(L_{3}-L_{4}\right)\left(L_{1}-L_{2}\right)\left(d_{m}+d_{c}\right)}{d^{2} \rho_{12} \rho_{34}} \\
-\frac{\left(L_{1}-L_{2}\right)\left(d_{m}+d_{c}\right)}{d \rho_{12}}-\frac{\left(L_{3}-L_{4}\right)\left(d_{m}+d_{c}\right)}{d \rho_{12} \rho_{34}} \\
\frac{1}{2} L_{3}+\frac{1}{2} L_{4}+\frac{\left(d_{m}+d_{c}\right)}{\rho_{34}}
\end{array}\right]
$$

The second part required for $U$ is as follows:

$$
m_{c} g_{0}\left(-\frac{\left(L_{1}-L_{2}\right)\left(d_{m}+d_{c}\right)}{d \rho_{12}}-\frac{\left(L_{3}-L_{4}\right)\left(d_{m}+d_{c}\right)}{d \rho_{12} \rho_{34}}\right)
$$

The robot's end effector is positioned at

$$
\left[\begin{array}{c}
\frac{1}{2} L_{1}+\frac{1}{2} L_{2}+\frac{d_{m}}{\rho_{12}}-\frac{\left(L_{3}-L_{4}\right)\left(L_{1}-L_{2}\right) d_{m}}{d^{2} \rho_{12} \rho_{34}}+\frac{\left(L_{1}-L_{2}\right) L_{5}}{d \rho_{12} \rho_{34}} \\
-\frac{\left(L_{1}-L_{2}\right) d_{m}}{d \rho_{12}}-\frac{\left(L_{3}-L_{4}\right) d_{m}}{d \rho_{12} \rho_{34}}+\frac{L_{5}}{\rho_{12} \rho_{34}} \\
\frac{1}{2} L_{3}+\frac{1}{2} L_{4}+\frac{\left(L_{3}-L_{4}\right) L_{5}}{d \rho_{34}}+\frac{d_{m}}{\rho_{34}}
\end{array}\right]
$$

and thus the third part required for $U$ is the second element from this vector. Combining this with Eq. (33) and (35) yields:

$$
\begin{aligned}
U & =m_{b} g_{0}\left(d_{m}+d_{b}\right) \frac{L_{2}-L_{1}}{d \rho_{12}} \\
& +m_{c} g_{0}\left(-\frac{\left(L_{1}-L_{2}\right)\left(d_{m}+d_{c}\right)}{d \rho_{12}}-\frac{\left(L_{3}-L_{4}\right)\left(d_{m}+d_{c}\right)}{d \rho_{12} \rho_{34}}\right) \\
& +m_{E} g_{0}\left(-\frac{\left(L_{1}-L_{2}\right) d_{m}}{d \rho_{12}}-\frac{\left(L_{3}-L_{4}\right) d_{m}}{d \rho_{12} \rho_{34}}+\frac{L_{5}}{\rho_{12} \rho_{34}}\right)
\end{aligned}
$$

Differentiating w.r.t the generalized coordinates $q$ yields:

$$
g(q)=\frac{\partial U}{\partial q}=\left[\begin{array}{c}
-\frac{\left(d_{c}+d_{m}\right) g_{0} m_{c}}{d \rho_{12}}-\frac{\left(d_{b}+d_{m}\right) g_{0} m_{b}}{d \rho_{12}}-\frac{d_{m} g_{0} m_{E}}{d \rho_{12}} \\
\frac{\left(d_{c}+d_{m}\right) g_{0} m_{c}}{d \rho_{12}}+\frac{\left(d_{b}+d_{m}\right) g_{0} m_{b}}{d \rho_{12}}+\frac{d_{m} g_{0} m_{E}}{d \rho_{12}} \\
-\frac{\left(d_{c}+d_{m}\right) g_{0} m_{c}}{d \rho_{12} \rho_{34}}-\frac{d_{m} g_{0} m_{E}}{d \rho_{12} \rho_{34}} \\
\frac{\left(d_{c}+d_{m}\right) g_{0} m_{c}}{d \rho_{12} \rho_{34}}+\frac{d_{m} g_{0} m_{E}}{d \rho_{12} \rho_{34}} \\
\frac{g_{0} m_{E}}{\rho_{12} \rho_{34}}
\end{array}\right]
$$


1) Working Space: Considering the data from paragraph II.A.3, the working volume of the end effector or the tool tip of the developed robot is limited to a $28 \times 28 \times 28 \mathrm{~mm}$ box. And the angular movement of the tool is limited to $\pm 1.2761 \mathrm{rad}, \pm 1.2761 \mathrm{rad}$ and $2 \pi \mathrm{rad}$ around $X, Y$ and $Z$ axes, respectively.

2) $3 D$ Variable RCM: Similar to RCM determination for a single joint, utilizing PCJM in 6DOF robot enables variable RCM in 3D space. Any virtual or physical points in space (excluding the singular configuration) can be defined as a pivoting point. During the motion this point is able to be moved. The importance of this specification for ophthalmic surgery is that the surgeon can move the eyeball by changing the position of RCM, during the surgery, and continue the operation. In conventional manual ophthalmic surgery this situation helps surgeons to see the different areas of retina through the microscope.

\section{G. Advantages of the Current Setup in Ophthalmic Surgery}

Summing up the mentioned points of the current robotic setup for ophthalmic surgery and comparing it with the similar systems, the following advantages are concluded:

Compatibility to OR: The dimensions and weight of the robot (measured: dimensions of $185 \times 44 \times 226 \mathrm{~mm}$ and a weight of $306 \mathrm{~g}$ ) make it a compatible and portable device for biomedical applications. Specifically, for eye surgery and micro vascular operations, this robot is suitable because it can be mounted on the organ which addresses a clinical challenge of tracking and compensating the patient's body motion.

Stiffness: The robot comprising PCJM enables highest possible stiffness against environmental disturbances.

Variable RCM: Definition of the desired RCM point is possible by this setup. It allows the surgeon to define and even move the pivoting point of the tool during the manipulation. Moving the organ without damaging the incision area is important in laprascopy and micro vascular operations when the surgeons are using incision points to introduce the surgical tools.

Safety: The piezo actuators used in this setup allow the surgeon to move the tool manually in the opposite direction of insertion by applying a force of around $5 N$.

\section{Performance VAlidation}

The precision of the tool tip motion, based on the visual observation from ophthalmic microscope, is estimated to be less than $5 \mu \mathrm{m}$ in $x$ and $y$ direction and $1 \mu \mathrm{m}$ in $z$ direction while the best human surgeon's precision recorded is $108 \mu \mathrm{m}$. The output forces of the robot's end effector were measured as $4.97 N$ in $x$ and $y$ and $2.84 N$ in $z$ directions. The maximum linear and angular velocity of the tool tip were measured as $67.46 \frac{\mathrm{mm}}{\mathrm{s}}$ and $86.91 \frac{\mathrm{deg} \text {. }}{\mathrm{s}}$, respectively.

\section{CONCLUSIONS AND FUTURE WORKS}

The design, for which already a patent is pending, and development of a micromanipulator for ophthalmic surgery has been described in this paper which can be also used for other micromanipulation applications. Joint elements of the robot comprising parallel piezo actuator pairs have been introduced which enables stiff and precise motion with sufficient output force. Furthermore, a serial configuration of these joints together with its simplified models has been shown. Finally, a brief report of the performance of the proposed mechanism in sense of precision, working volume, physical dimensions and output forces has been mentioned.

\section{REFERENCES}

[1] S. Rogers et al., "The prevalence of retinal vein occlusion: pooled data from population studies from the US, Europe, Asia, and Australia," in Ophthalmology, vol. 117, no. 2, pp. 313-319, Feb. 2010

[2] L. Bynoe et al., "Retinal endovascular surgery for central retinal vein occlusion: initial experience of four surgeons," in Retina, Coral Springs, FL, USA, vol. 25, issue 5, pp. 625-632, Jul.-Aug. 2005

[3] W. Wei et al., "Enabling technology for microvascular stenting in ophthalmic surgery," in American Society of Mechanical Engineers (ASME): Journal of Medical Devices, vol 4, no. 1, Mar. 2010

[4] S. Sinch and C. Riviere, "Physiological tremor amplitude during retinal microsurgery," in Proceedings of the 28th IEEE Northeast Bioengineering Conference, PA, USA, pp. 171-172, Apr. 2002

[5] A. Guerrouad and D. Jolly, "Automatic analysis of weariness during a micromanipulation task by SMOS," in Proceedings of the IEEE Engineering in Medicine \& Biology Society 11th Annual International Conference, Seattle, DC, USA, pp. 906-907, Nov. 1989

[6] S. Charles et al., "Dexterity-enhanced Telerobotic Microsurgery," in Proceedings of the IEEE International Conference on Advanced Robotics, Monterey, CA, USA, pp.5-10, Jul. 1997

[7] W. Wei et al., "Design and Theoretical Evaluation of Micro-Surgical Manipulators for Orbital Manipulation and Intraocular Dexterity," in Proceedings of the IEEE International Conference on Robotics and Automation, Roma, Italy, pp. 3389-3395, Apr. 2007

[8] W.T. Ang et al., "Active tremor compensation in microsurgery," in Proceedings of the 26th Annual International Conference of the IEEE Engineering in Medicine and Biology Society, Pittsburg, PA, USA, pp. 2738-2741, Sep. 2004

[9] A. Uneri et al., "New steady-hand eye robot with micro-force sensing for vitreoretinal surgery," in Proceedings of IEEE RAS EMBS International Conference on Biomedical Robotics and Biomechatronics, Tokyo, Japan, pp. 814-819, Sep. 2010

[10] T. Ueta et al., "Robot-assisted vitreoretinal surgery: Development of a prototype and feasibility studies in an animal model," in Ophthalmology, vol. 116, issue 8, pp. 1538-1543, 2009

[11] P. Caers et al., "Precision experiments on a comanipulated robotic system for use in retinal surgery," in Proceedings of the 2011 SCATh Joint Workshop on New Technologies for Computer/Robot Assisted Surgery, Graz, Austria, pp. 1-7, Jul. 2011

[12] T. Meenink, "Vitreo-Retinal eye surgery robot: sustainable precision," $\mathrm{PhD}$ thesis, TU Eindhoven, Eindhoven, The Netherlands, ISBN:97890-386-2800-4, 2011

[13] M. A. Nasseri et al., "Clinical motion tracking and motion analysis during ophthalmic surgery using electromagnetic tracking system," in Proceedings of the 5th International IEEE Conference on BioMedical Engineering and Informatics (BMEI), Chongqing, China, pp. 10581062 , Oct. 2012

[14] L. Romdhane, "Design and analysis of a serial-parallel manipulator," In Mechanism and Machine Theory, vol. 34, issue 7, pp. 1037-1055, Oct. 1999

[15] T. K. Tanev, "Kinematics of a hybrid (parallel-serial) robot manipulator," In Mechanism and Machine Theory, vol. 35, pp. 1183-1196, 2000

[16] G. Yang, C. Weihai and E. H. L. Ho, "Design and Kinematics Analysis of a Modular Hybrid Parallel-Serial Manipulator," In 7th International Conference on Control, Automation, Robotics and Vision (ICARCV), vol. 1, pp. 45-50, Dec. 2002

[17] SmarAct GmbH, "SmarAct perfect motion. Product Catalogue," SmarAct GmbH, Oldenburg, Germany, vol. 12, pp. 26-27, 2013

[18] J. Denavit and R.S. Hartenberg, "A kinematic notation for lower pair mechanisms based on matrices," in Transaction of the American Society of Mechanical Engineers (ASME). Journal of Applied Mechanics, vol. 77 , pp. $215-221,1955$ 